\title{
A Pilot Multicenter Study Evaluating the Expression of p53 and ki-67 in Gastric Tumors and Their Utility for Guiding Treatment Strategy
}

\author{
Il Hyun Baek ${ }^{1}$, Kyoung Oh Kim², Jeong Won Kim³ ${ }^{3}$ Kyeong Won Min ${ }^{4}$
}

Corresponding author:

II Hyun Baek, M.D., PhD

Eulji University Hospital

1306, Dunsan-dong, Seo-gu

Daejeon, Rep. of KOREA 35233

Tel.: + 82-42-611-3063

E-mail: drandrea100@daum.net
Department of Gastroenterology

Eulji University School of Medicine

${ }^{1}$ Department of Gastroenterology, Eulji University Hospital

Eulji University School of Medicine, Daejeon, Korea

${ }^{2}$ Department of Gastroenterology, Hallym University Sacred Heart Hospital

Hallym University College of Medicine, Anyang, Korea

${ }^{3}$ Department of Pathology, Kangnam Sacred Heart Hospital

Hallym University College of Medicine, Seoul, Korea

${ }^{4}$ Department of Human Resource Development, Graduate School of Chung-Ang University Seoul, Korea

\section{ABSTRACT}

Background: p53 mutation is the most common genetic alteration in cancers and influences clinical progression. Ki-67 protein is a cellular marker for proliferation in cancer or premalignant lesion. The aim of this study is to investigate whether p53 and Ki-67 measurements in gastric tumors would be helpful in determining treatment strategy.

Methods: Immunohistochemical staining using monoclonal antibodies to p53 and Ki-67 was performed on specimens from 29 gastric adenomas (GA) by endoscopic submucosal dissection (ESD) and 240 gastric cancers (GC) by ESD or gastrectomy. Tumor cells with nuclear p53 and Ki-67 protein expression were arbitrarily graded into four groups: $<10 \%=$ negative, $10-30 \%=1+, 30-60 \%=2+$, and $>60 \%=3+$.

Results: The mean tumor sizes in the GA and GC groups were $17.3 \pm 11.4 \mathrm{~mm}$ and $32.0 \pm$ $20.9 \mathrm{~mm}$ respectively $(P<0.001)$. p53 positivity was not different between the GA and GC groups $(P=0.149)$, but Ki-67 positivity was significantly different between the 2 groups $(P=$ 0.001). In addition, Ki-67 positivity tended to be increased as the pathologic progression changed from adenoma to cancer.

Conclusions: Ki-67 positivity grade seems to be correlated with malignancy potential. Even if endoscopic biopsy showed low grade dysplasia, in lesions with high Ki-67 positivity, it is better to consider active ESD rather than just long-term follow up.

Key words: Gastric adenoma, Gastric cancer, Immunohistochemistry, p53, Ki-67.

\section{INTRODUCTION}

Received: 08.12.2017 Accepted: 16.12.2017
The stomach adenoma is a common benign tumor originating from the gastric glandular epithelium, accounting for $10 \%$ of all stomach polyps (1). Gastric adenoma is also considered a precancerous lesion $(2,3)$. The risk factors for malignancy in gastric adenomas are large tumor size and high-grade 
dysplasia (4-6). However, these factors are not sufficient to predict the cancerous progression of each adenoma.

The p53 tumor suppressor gene functions by regulating cell proliferation through control of the G1 arrest checkpoint in the cell cycle $(7,8)$. Mutation in the p53 gene is one of the most common genetic lesions associated with gastric cancer, and some studies have reported that the expression of $p 53$ is related to bad prognosis (9-14).

Ki-67 is related to cell proliferation $(15,16)$, and advanced gastric cancer (AGC) patients have higher Ki-67 labeling rates than early gastric cancer patients. Also, Ki-67 labeling index was significantly higher in gastric cancer patients with lymph node metastasis $(17,18)$.

Although there are some qualitative studies for $\mathrm{p} 53$ and Ki-67 expression using immunohistochemistry $(\mathrm{IHC})$ in gastric adenoma and gastric cancer, there is no report on the quantification of expression intensity. We wanted to know whether immunostaining grading of p53 or Ki-67 of tumor cells is different or not between gastric cancer and gastric low-grade adenoma. We divided patients with gastric adenoma or gastric cancer who were undergoing ESD or gastrectomy into 4 groups, depending on the expression proportion of p53 and $\mathrm{Ki}-67$ by using $\mathrm{IHC}$, and investigated whether this semi-quantified expression index for p53 and Ki-67 are possible indicators with regard to the prognosis of gastric adenoma.

\section{METHODS}

\section{Patients}

We analyzed p53 and Ki-67 expression in the tumor tissue of 29 gastric adenoma with low-grade dysplasia patients who were treated by endoscopic submucosal dissection (GA-LGD group) and 240 gastric cancer patients who were treated by endoscopic submucosal dissection or gastrectomy (GC group) at Kangnam Sacred Heart Hospital, Hallym University Sacred Heart Hospital of Hallym University, and Eulgi University Hospital from January 2009 to December 2016. The ethics committee of Hallym University Sacred Heart Hospital approved the research protocol and received informed consent from each patient (Clinical trial registration No. KCT 0002314).

\section{Methods}

Paraffin-embedded samples were cut into fourmicrometer sections and mounted on poly-L-lysine coated slides. The specimens were processed by an automated IHC system (BondTM System, Leica Microsystems, Bannockburn, IL, U.S.A.) using monoclonal antibodies against p53 (DO-7, mouse monoclonal, Dako, Glostrup, Denmark, dilution 1: 3000) and Ki-67 (MIB-5, mouse monoclonal, Dako, dilution 1: 250). The proportion of tumor cells with nuclear p53 protein immunostaining was arbitrarily graded into four groups: $<10 \%=$ negative, $10-30 \%=1+, 30-60 \%=2+$, and $>60 \%=3+$ of the total tumour cell population per section (fig. 1). Four sections from each tumor were scored. A tumor was scored as p53 positive when at least $10 \%$ of the tumor cells showed nuclear p53 protein accumulation. The Ki-67 labeling index was obtained by using light microscopy at $400 \times$ magnification and the ratio was calculated as the percentage of 500 tumor cells, and also semi-quantitatively graded from negative to $3+$ in the same manner (fig. 2). One pathologist in each hospital evaluated specimens after consensus meeting.

\section{Statistical analysis}

The Mann-Whitney $U$ test was used to compare age and lesion size. When comparing IHC staining between

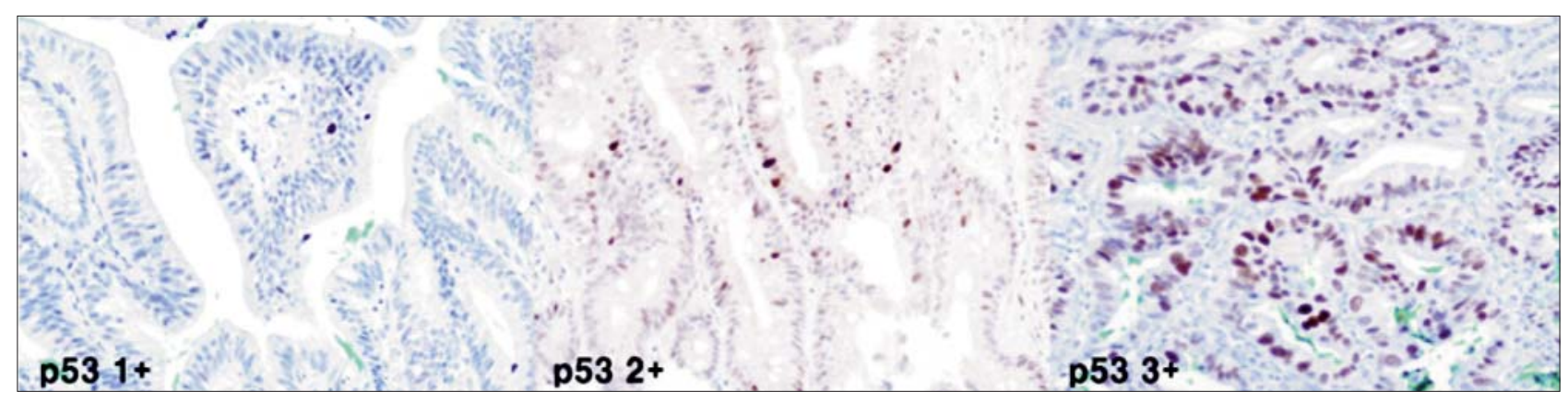

Figure 1 - The proportion of tumor cells with nuclear p53 protein immunostaining was arbitrarily graded into four groups: $<10 \%=$ negative, $10-30 \%=1+, 30-60 \%=2+$, and $>60 \%=3+$ of the total tumor cell population per section. 


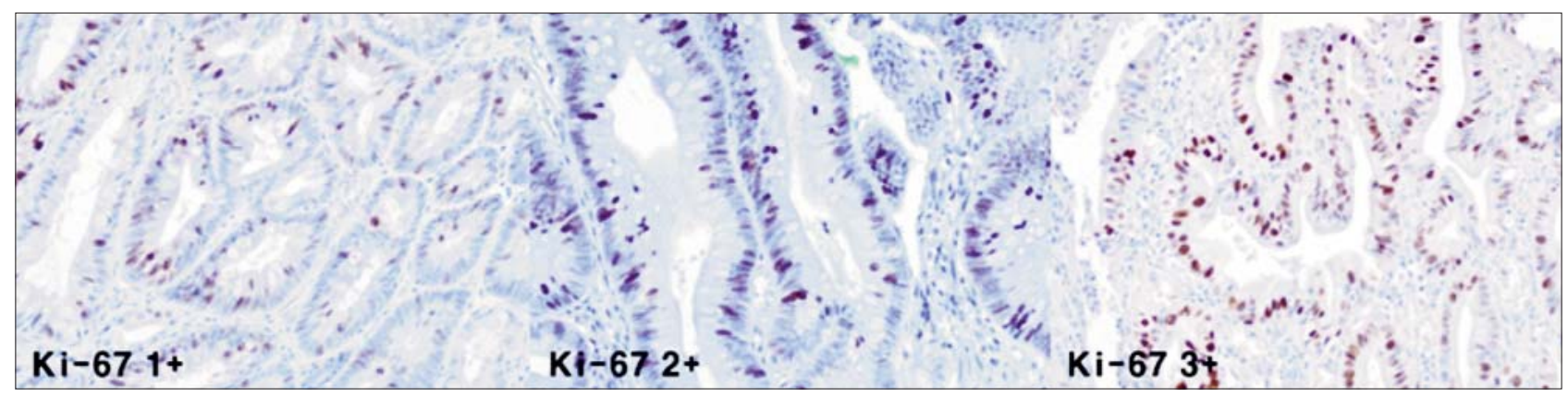

Figure 2 - The Ki-67 labeling index was obtained by using light microscopy at $400 \mathrm{x}$ magnification and the ratio was calculated as the percentage of $\mathbf{5 0 0}$ tumor cells, and also semi-quantitatively graded from negative to $3+$ in the same manner.

gastric cancer and gastric adenoma, linear by linear associations and Pearsons' chi-square test were performed. Values were considered significant at $p<0.05$. All analysis was performed using PASW Statistics for Windows, Version 18.0 (SPSS, Inc., Chicago, IL).

\section{RESULTS}

There were 29 cases of gastric adenoma with low grade dysplasia and 240 cases with advanced gastric cancer (AGC) and early gastric cancer (EGC). The mean age was $63.5 \pm 11.8$ years and the mean tumor size was $30.4 \pm 20.6 \mathrm{~mm}$. Comparing the two groups, the mean tumor size was $17.3 \pm 11.4 \mathrm{~mm}$ in the GA-LGD group and $32.0 \pm 20.9 \mathrm{~mm}$ in the GC group while the mean age was $64.7 \pm 9.8$ years old in the GA-LGD group and $63.4 \pm 12.1$ years old in the GC group. There was no significant difference between the two groups when comparing these parameters. The clinicopathologic characteristics of the GA-LGD and GC groups are shown in table 1.

In the GA-LGD group, the expression index for p53 was as follows: negative, $10.3 \% ; 1+, 31.0 \% ; 2+$, $55.2 \%$; and $3+, 3.4 \%$. In the GC group, the expression index was: negative, 32.5\%; 1+, 10.0\%; 2+, 56.7\%; and $3+, 0.8 \%$ (table 2 ). The semi-quantified positivity of 553 was not significantly different between the two groups ( $p=0.149$ ). For the GA-LGD group, the expression index of Ki-67 was as follows: negative, $0.0 \% ; 1+, 13.8 \% ; 2+, 27.6 \%$; and $3+, 58.6 \%$. For the GC group, the expression index was: negative, $0.4 \%$; $1+, 5.4 \% ; 2+, 7.1 \%$; and $3+, 87.1 \%$. The semiquantified positivity for Ki-67 was significantly higher in the GC group $(p=0.001)$. In addition, the semiquantified positivity for Ki-67 increased as the tumor changed from adenoma to cancer (table 2, fig. 3).

The semi-quantified positivity of p53 was not
Table 1 - Baseline characteristics.

\begin{tabular}{lcccc}
\hline & GA-LGD $(\mathbf{n = 2 9})$ & GC $(\mathbf{n = 2 4 0 )}$ & Total $(\mathbf{n = 2 6 9})$ & $\mathbf{p}$ value \\
\hline Gender & & & & 0.119 \\
\hline Male & 24 & 165 & 189 & \\
\hline Female & 5 & 75 & 80 & \\
\hline Age & $64.7 \pm 9.8$ & $63.4 \pm 12.1$ & $63.5 \pm 11.8$ & 0.664 \\
Tumor size $(\mathrm{mm})$ & $17.3 \pm 11.4$ & $32.0 \pm 20.9$ & $30.4 \pm 20.6$ & $<0.001$ \\
\hline
\end{tabular}

GA-LGD, gastric adenoma with low grade dysplasia; GC, Gastric cancer

\begin{tabular}{|c|c|c|c|c|}
\hline & Expression & GA-LGD $(n=29)$ & $\mathrm{GC}(n=240)$ & $p$ value \\
\hline \multirow[t]{4}{*}{ p53 } & Negative & $3(10.3 \%)$ & $78(32.5 \%)$ & 0.149 \\
\hline & $1+$ & $9(31.0 \%)$ & $24(10.0 \%)$ & \\
\hline & $2+$ & $16(55.2 \%)$ & $136(56.7 \%)$ & \\
\hline & $3+$ & $1(3.4 \%)$ & $2(0.8 \%)$ & \\
\hline \multirow[t]{4}{*}{ Ki-67 } & Negative & $0(0.0 \%)$ & $1(0.4 \%)$ & 0.001 \\
\hline & $1+$ & $4(13.8 \%)$ & $13(5.4 \%)$ & \\
\hline & $2+$ & $8(27.6 \%)$ & $17(7.1 \%)$ & \\
\hline & $3+$ & $17(58.6 \%)$ & $209(87.1 \%)$ & \\
\hline
\end{tabular}

GA-LGD, gastric adenoma with low grade dysplasia; GC, Gastric cancer.
Table 2 - Semi-quantitative in $\mathrm{p53}$ and $\mathrm{Ki}-67$ grade of expression intensity 

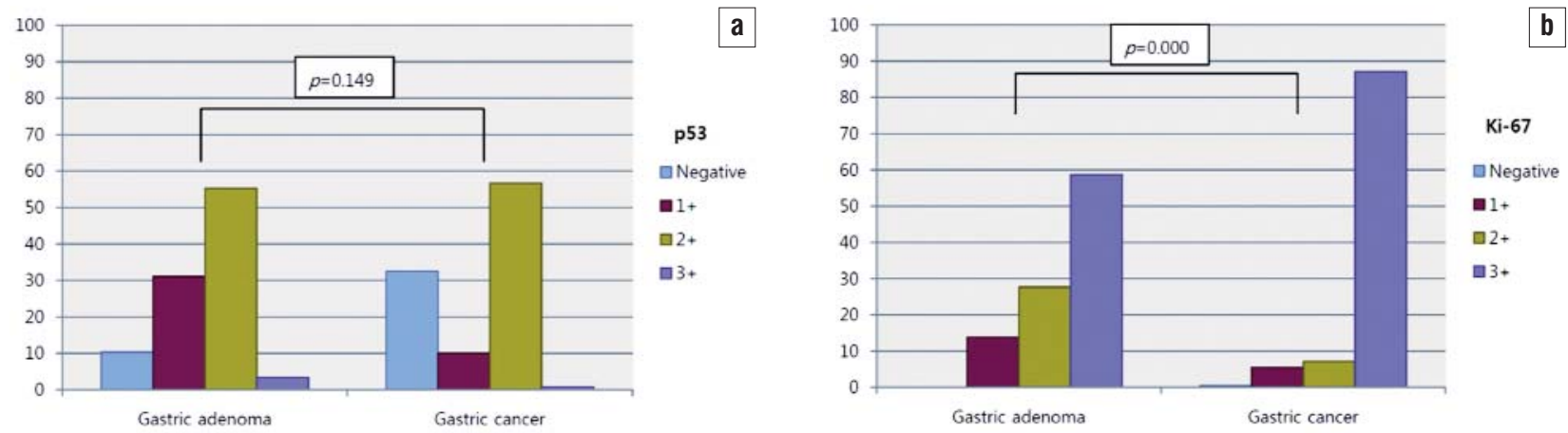

Figure 3 - The semi-quantified positivity of p53 was not significantly different between the two groups (a). The semi-quantified positivity for KI-67 increased as the tumor changed from adenoma to cancer (b).

significantly different according to the tumor pathology $(p=0.777)$. The semi-quantified positivity for Ki-67 was not significantly different according to the tumor pathology ( $p=0.101$ ) (table 3).

The semi-quantified positivity of p53 was significantly different according to $T$ stage $(p=0.047)$. The semi-quantified positivity for Ki-67 was significantly different according to T stage $(p=0.013)$. The over $2+$ positivity for p53 increased as the tumor changed from
T0 to T4. 3+ positivity for Ki-67 increased as the tumor changed from T0 to T4 (table 4).

The semi-quantified positivity of $\mathrm{p} 53$ was significantly different according to lymph node status ( $p=0.047)$. The semi-quantified positivity for Ki-67 was significantly different according to lymph node status $(p=0.027)$. The over 2+ positivity for p53 increased as the tumor changed from NO to N3. 3+ positivity for Ki-67 increased as the tumor changed from NO to N3 (table 5).

Table 3 - Semi-quantitative grade of expression intensity in p53 and Ki-67 by pathology among gastric cancer patients $(n=240)$

\begin{tabular}{|c|c|c|c|c|c|c|}
\hline & Expression & WD adenoca & MD adnoca & PD adenoca & Signet ring cell carcinoma & p value * \\
\hline \multirow[t]{4}{*}{$\mathrm{p} 53$} & Negative & $20(32.3)$ & $26(33.8)$ & $17(25.8)$ & $15(42.9)$ & 0.777 \\
\hline & $1+$ & $7(11.3)$ & $7(9.1)$ & $7(10.6)$ & $3(8.6)$ & \\
\hline & $2+$ & $34(54.8)$ & $44(57.1)$ & $42(63.6)$ & $16(45.7)$ & \\
\hline & $3+$ & $1(1.6)$ & $0(0.0)$ & $0(0.0)$ & $1(2.9)$ & \\
\hline \multirow[t]{4}{*}{ Ki-67 } & Negative & $1(1.6)$ & $0(0.0)$ & $0(0.0)$ & $0(0.0)$ & 0.101 \\
\hline & $1+$ & $2(3.2)$ & $3(3.9)$ & $3(4.5)$ & $5(14.3)$ & \\
\hline & $2+$ & $5(8.1)$ & $3(3.9)$ & $4(6.1)$ & $5(14.3)$ & \\
\hline & $3+$ & $54(87.1)$ & $71(92.2)$ & $59(89.4)$ & $25(71.4)$ & \\
\hline
\end{tabular}

* Linear by linear association was used.

The data are shown as numbers (\%).

WD, well diffrentiated; MD, moderate diffrentiated; PD, poorly differentiated; adenoca, adenocarcinoma

Table 4 - Semi-quantitative grade of expression intensity in p53 and Ki-67 by the depth of invasion among gastric cancer patients $(n=240)$

\begin{tabular}{|c|c|c|c|c|c|c|c|}
\hline & Expression & TO & $\mathrm{T} 1$ & T2 & T3 & T4 & p value * \\
\hline \multirow[t]{4}{*}{ p53 } & Negative & 7 (53.8) & $46(33.3)$ & $6(33.3)$ & $12(23.1)$ & $6(35.3)$ & 0.047 \\
\hline & $1+$ & $1(7.7)$ & $18(13.0)$ & $2(11.1)$ & $3(5.8)$ & $0(0.0)$ & \\
\hline & $2+$ & $5(38.5)$ & $73(52.9)$ & $10(55.6)$ & $36(69.2)$ & $11(64.7)$ & \\
\hline & $3+$ & $0(0.0)$ & $1(0.7)$ & $0(0.0)$ & $1(1.9)$ & $0(0.0)$ & \\
\hline \multirow[t]{4}{*}{$\mathrm{Ki}-67$} & Negative & $1(7.7)$ & $0(0.0)$ & $0(0.0)$ & $0(0.0)$ & $0(0.0)$ & 0.013 \\
\hline & $1+$ & $1(7.7)$ & $10(7.2)$ & $0(0.0)$ & $2(3.8)$ & $0(0.0)$ & \\
\hline & $2+$ & $1(7.7)$ & $11(8.0)$ & $4(22.2)$ & $1(1.9)$ & $0(0.0)$ & \\
\hline & $3+$ & $10(76.9)$ & $117(84.8)$ & $14(77.8)$ & $49(94.2)$ & $17(100.0)$ & \\
\hline
\end{tabular}

* Linear by linear association was used.

The data of two patients could not be accessed.

The data are shown as numbers (\%). 
Table 5 - Semi-quantitative grade of expression intensity in p53 and Ki-67 by lymph node status among gastric cancer patients $(\mathrm{n}=\mathbf{2 4 0})$

\begin{tabular}{|c|c|c|c|c|c|c|}
\hline & Expression & NO & N1 & N2 & N3 & p value* \\
\hline \multirow[t]{4}{*}{ p53 } & Negative & $60(34.5)$ & $9(33.3)$ & $4(28.6)$ & $4(17.4)$ & 0.047 \\
\hline & $1+$ & $21(12.1)$ & $1(3.7)$ & $0(0.0)$ & $2(8.7)$ & \\
\hline & $2+$ & $92(52.9)$ & $16(59.3)$ & $10(71.4)$ & $17(73.9)$ & \\
\hline & $3+$ & $1(0.6)$ & $1(3.7)$ & $0(0.0)$ & $0(0.0)$ & \\
\hline \multirow[t]{4}{*}{$\mathrm{Ki}-67$} & Negative & $1(0.6)$ & $0(0.0)$ & $0(0.0)$ & $0(0.0)$ & 0.027 \\
\hline & $1+$ & $12(6.9)$ & $1(3.7)$ & $0(0.0)$ & $0(0.0)$ & \\
\hline & $2+$ & $15(8.6)$ & $1(3.7)$ & $0(0.0)$ & $1(4.3)$ & \\
\hline & $3+$ & $146(83.9)$ & $25(92.6)$ & $14(100.0)$ & $22(95.7)$ & \\
\hline
\end{tabular}

* Linear by linear association was used.

The data of two patients could not be accessed.

The data are shown as numbers (\%).

The semi-quantified positivity of p53 was significantly different according to stage $(p=0.009)$. The semiquantified positivity for Ki-67 was significantly different according to stage $(p=0.005)$. The over $2+$ positivity for p53 increased as the tumor changed from stage 0 to stage 4. 3+ positivity for Ki-67 increased as the tumor changed from stage 0 to stage 4 (table 6).

\section{DISCUSSION}

The gastric adenoma undergoes malignant transformation that gradually changes from low-grade to highgrade dysplasia. Low-grade dysplastic gastric adenomas evolved into stomach cancer after an average of 48 months (range 21-85) of follow-up observation (19). High-grade dysplastic gastric adenomas progress to cancer at $60-70 \%$ and progress to stomach cancer at 2$6 \%$ per year $(5,19,20)$. The $p 53$ tumor suppressor gene is located on chromosome 17p and regulates cell cycle, DNA repair and synthesis, and apoptosis $(21,22)$. The production of p53 increases with intracellular damage or DNA damage, and p53 induces cell cycle arrest at the
G1 / S-junction (7). The loss of $\mathrm{p} 53$ function is related to the progression of the tumor by allowing cells with damaged DNA to continue to multiply (23). In some studies, p53 expression in stomach cancer has been reported to be bad prognosis (9-14), but other reports have shown that p53 expression has no effect on clinical results (24-26). The reasons for these conflicting results are unclear. However, recent studies have shown a correlation between p53 expression and higher proliferative activity; suggesting that changes in the p53 gene promote cell proliferation and uncontrolled cell division $(22,27,28)$. A comparison of stomach cancer and gastric adenoma has shown that stomach cancer has higher incidence of p53 than gastric adenoma $(29,30)$. Our results do not show statistically significant differences on the expression of p53 between gastric adenoma and gastric cancer. Moreover, Oiwa et al. (31) reported that only $20 \%$ of EGC patients had p53 overexpression. Uchino et al. (32) reported the accumulation of p53 protein was observed in 15 (37\%) of 41 cohesive type EGC patients and was not seen in 18 patients with signet ring cell type EGC. In this study, the

Table 6 - Semi-quantitative grade of expression intensity in p53 and Ki-67 by stage among gastric cancer patients $(n=240)$

\begin{tabular}{|c|c|c|c|c|c|c|c|}
\hline & Expression & Stage 0 & Stage 1 & Stage 2 & Stage 3 & Stage 4 & $p$ value * \\
\hline \multirow[t]{4}{*}{ p53 } & Negative & 7 (53.8) & $50(33.6)$ & $12(30.8)$ & $8(24.2)$ & $0(0.0)$ & 0.009 \\
\hline & $1+$ & $1(7.7)$ & $20(13.4)$ & $1(2.6)$ & $2(6.1)$ & $0(0.0)$ & \\
\hline & $2+$ & $5(38.5)$ & $78(52.3)$ & $25(64.1)$ & $23(69.7)$ & $4(100.0)$ & \\
\hline & $3+$ & $0(0.0)$ & $1(0.7)$ & $1(2.6)$ & $0(0.0)$ & $0(0.0)$ & \\
\hline \multirow[t]{4}{*}{$\mathrm{Ki}-67$} & Negative & $1(7.7)$ & $0(0.0)$ & $0(0.0)$ & $0(0.0)$ & $0(0.0)$ & 0.005 \\
\hline & $1+$ & $1(7.7)$ & $10(6.7)$ & $2(5.1)$ & $0(0.0)$ & $0(0.0)$ & \\
\hline & $2+$ & $1(7.7)$ & $15(10.1)$ & $0(0.0)$ & $1(3.0)$ & $0(0.0)$ & \\
\hline & $3+$ & $10(76.9)$ & 124 (83.2) & $37(94.9)$ & $32(97.0)$ & $4(100.0)$ & \\
\hline
\end{tabular}

* Linear by linear association was used.

The data of two patients could not be accessed.

The data are shown as numbers (\%). 
semi-quantified positivity of p53 and Ki-67 were not significantly different according to the tumor pathology $(p=0.777, p=0.101$, respectively). Ki-67 monoclonal antibody reacts with a nuclear antigen that exists in proliferating cells throughout the cell cycle (late G1, $S-, G 2$, and M-phase) but not in G0 phase cells (33). The Ki-67 marker index was higher in patients with AGC than in EGC patients and was reported to be a useful predictor of lymph node metastasis and invasion depth (17). In this study, the semi-quantified positivity of p53 and Ki-67 were significantly different according to $T$ stage ( $p=0.047, p=0.013$, respectively) and increased as the tumor changed from TO to T4. The semi-quantified positivity of p53 and Ki-67 were significantly different according to lymph node status $(p=0.047, p=0.027$, respectively) and increased as the tumor changed from NO to N3. The semi-quantified positivity of p53 and Ki-67 were significantly different according to lymph node status ( $p=0.047, p=0.027$, respectively) and increased as the tumor changed from NO to N3. The semi-quantified positivity of p53 and Ki-67 were significantly different according to stage $(p=0.009, p=0.005$, respectively) and increased as the tumor changed from stage 0 to stage 4 . In another study, p53 and Ki-67 expression were related to response to chemotherapy (33-35). Additionally, our study shows that the accumulation intensity of the Ki- 67 protein tends to be increased as tumors progress from gastric adenoma to gastric cancer. The results of this study seem to be helpful in determining whether Ki-67 IHC analysis is help in the decision of ESD in gastric adenoma with low grade dysplasia.

A limitation of this study is the lack of information regarding the follow-up of patients with gastric adenoma with low-grade dysplasia, because this is a pilot study. In the future, a well-designed long-term follow-up study is needed to estimate the effect of $\mathrm{Ki}-67$ on low-grade gastric adenoma.

\section{CONCLUSIONS}

Ki-67 positivity grade seems to be correlated with malignancy potential. Even if endoscopic biopsy showed low grade dysplasia, in lesions with high Ki-67 positivity, it is better to consider active ESD rather than just long-term follow up.

\section{Conflict of interest}

The authors declare that there is no conflict of interest.

\section{REFERENCES}

1. Ming SC. The classification and significance of gastric polyps. Monogr Pathol. 1977;18:149-75.

2. Morson BC, Sobin LH, Grundmann E, Johansen A, Nagayo T, SerckHanssen A. Precancerous conditions and epithelial dysplasia in the stomach. J Clin Pathol. 1980;33:711-21.

3. Correa P. Precursors of gastric and esophageal cancer. Cancer. 1982;50(11 Suppl):2554-65.

4. Johansen A. Elevated early gastric carcinoma. differential diagnosis as regards adenomatous polyps. Pathol Res Pract. 1979;164:316-30.

5. Kamiya T, Morishita T, Asakura H, Miura S, Munakata Y, Tsuchiya M. Long-term follow-up study on gastric adenoma and its relation to gastric protruded carcinoma. Cancer. 1982;50:2496-503.

6. Kolodziejczyk P, Yao T, Oya M, et al. Long-term follow-up study of patients with gastric adenomas with malignant transformation. An immunohistochemical and histochemical analysis. Cancer. 1994;74:2896-907.

7. Fenoglio-Preiser CM, Wang J, Stemmermann GN, Noffsinger A. TP53 and gastric carcinoma: a review. Hum Mutat. 2003;21:258-70.

8. Wiksten JP, Lundin J, Nordling S, Kokkola A, von Boguslawski K, Haglund $\mathrm{C}$. The prognostic value of p27 in gastric cancer. Oncology. 2002;63:180-4.

9. Martin HM, Filipe MI, Morris RW, Lane DP, Silvestre F. P53 expression and prognosis in gastric carcinoma. Int J Cancer. 1992;50:859-62.

10. Joypaul BV, Hopwood D, Newman EL, et al. The prognostic significance of the accumulation of p53 tumour-suppressor gene protein in gastric adenocarcinoma. Br J Cancer. 1994;69:943-6.

11. Maehara Y, Okuyama T, Kakeji Y, Endo K, Yamamoto M, Sugimachi K. A tumour-associated cell-surface glycoprotein accompanying p53 overexpression and higher growth potential for gastric cancer. $\mathrm{Br} \mathrm{J}$ Cancer. 1995;71:999-1002.

12. Monig SP, Eidt S, Zirbes TK, Stippel D, Baldus SE, Pichlmaier H. P53 expression in gastric cancer: Clinicopathological correlation and prognostic significance. Dig Dis Sci. 1997;42:2463-7.

13. Maehara Y, Tomoda M, Hasuda S, et al. Prognostic value of p53 protein expression for patients with gastric cancer--a multivariate analysis. Br J Cancer. 1999;79:1255-61.

14. Lee HK, Lee HS, Yang HK, et al. Prognostic significance of bcl-2 and p53 expression in gastric cancer. Int J Colorectal Dis. 2003;18:518-25.

15. Noh SH, Yoo CH, Kim HG, et al. Prognostic significance of p53, cerbB-2, nm23 and Ki-67 expression in patients with advanced gastric carcinoma. J Korean Cancer Assoc. 1999;31:699-709.

16. Oh YL, Han JH, Ko YH, Park CK, Ree HJ. Correlation between p53 immunohistochemical expression, DNA ploidy and Ki-67 expression in gastric carcinoma. Korean J Pathol. 1997;31:1264-71.

17. Kakeji Y, Korenaga D, Tsujitani S, Haraguchi M, Maehara Y, Sugimachi K. Predictive value of Ki-67 and argyrophilic nucleolar organizer region staining for lymph node metastasis in gastric cancer. Cancer Res. 1991:51:3503-06.

18. Igarashi N, Takahashi M, Ohkubo H, Omata K, lida R, Fujimoto S. Predictive value of Ki-67, p53 protein, and DNA content in the diagnosis of gastric carcinoma. Cancer. 1999;86:1449-54.

19. M Rugge, M Cassaro, F Di Mario, et al. The long term outcome of gastric non-invasive neoplasia. Gut. 2003;52:1111-6.

20. de Vries AC, van Grieken NC, Looman CW, et al. Gastric cancer risk in patients with premalignant gastric lesions: A nationwide cohort study in the Netherlands. Gastroenterology. 2008; 134:945-52.

21. Hollstein MC, Sidranslcy D, Vigelstein B, Harris CC. p53 mutations in human cancers. Science. 1991;253:49-50.

22. Levine AJ. The p53 tumor-suppressor gene. N Engl J Med. 1992;326:1350-2

23. Greenblatt MS, Bennett WP, Hollstein M, Harris CC. Mutations in the p53 tumor suppressor gene: Clues to cancer etiology and molecular pathogenesis. Cancer Res. 1994;54:4855-78.

24. Motojima K, Furui J, Kohara N, Kanematsu T. Expression of p53 protein in gastric carcinomas is not independently prognostic. Surgery. 1994;116:890-5.

25. Schneider BG, Hilsenbeck SG, Hensel CH, et al. p53 mutations in 
gastric and colorectal cancers in Texas Hispanics versus Anglos. Virchows Archiv. 1994;424:187-93.

26. Gabbert HE, Muller W, Schneiders A, Meier S, Hommel G. The relationship of $p 53$ expression to the prognosis of 418 patients with gastric carcinoma. Cancer. 1995;76:720-6.

27. Ishida $\mathrm{H}$, Irie $\mathrm{K}$, Itoh T, Furukawa $\mathrm{T}$, Tokunaga 0 . The prognostic significance of p53 and bcl-2 expression in lung adenocarcinoma and its correlation with Ki-67 growth fraction. Cancer. 1997;80:1034-45.

28. Aizawa K, Ueki K, Suzuki S, et al. Apoptosis and Bbcl-2 expression in gastric carcinomas: Correlation with clinicopathological variables, p53 expression, cell proliferation and prognosis. Int J Oncol. 1999;14:8591.

29. Shiao YH, Rugge M, Correa P, Lehmann HP, Scheer WD. P53 alteration in gastric precancerous lesions. Am J Pathol. 1994;144:511-17.

30. Lee DS, Kang SB, Baek JT, et al. Immunohistochemical expression of bcl-2, bcl-xL, bax, p53 proteins in gastric adenoma and adenocarcinoma. Korean J Gastroenterol. 2005;45:394-400.
31. Uchino S, Noguchi M, Ochiai A, et al. P53 mutation in gastric cancer: A genetic model for carcinogenesis is common to gastric and colorectal cancer. Int J Cancer. 1993;54:759-64.

32. 0 iwa H, Maehara $\mathrm{Y}$, Ohno S, Saito T, Kobayashi M, Hirohashi S. Growth pattern and p53 overexpression in patients with early gastric cancer. Cancer. 1995;75:1454-9.

33. Gerdes J, Lemke H, Baisch H, Wacker HH, Schwab U, Stein H. Cell cycle analysis of a cell proliferation-associated human nuclear antigen defined by the monoclonal antibody Ki-67. J Immunol. 1984; 133:1710-5.

34. Yonemura Y, Ooyama S, Sugiyama K, et al. Growth fractions in gastric carcinomas determined with monoclonal antibody Ki-67. Cancer. 1990;65:1130-4.

35. Hosaka N, Ichikawa Y, Ishikawa T, et al. Correlation of immunohistochemical p53 labeling index with inhibition rate in chemosensitivity test in gastric and colon cancer. Anticancer Res. 2001;21:229-35. 